\title{
DISCRIMINATION BETWEEN HEALTY SUBJECTS AND PATIENTS WITH PULMONARY EMPHYSEMA BY DETECTION OF ABNORMAL RESPIRATION
}

\author{
Masaru Yamashita, Shoichi Matsunaga and Sueharu Miyahara \\ Department of Computer and Information Sciences, Nagasaki University, JAPAN
}

\begin{abstract}
In this paper, we propose a robust classification strategy for distinguishing between a healthy subject and a patient with pulmonary emphysema on the basis of lung sounds. A symptom of pulmonary emphysema is that almost all lung sounds include some abnormal (i.e., adventitious) sounds. However, the great variety of possible adventitious sounds and noises at auscultation makes high-accuracy detection difficult. To overcome this difficulty, our strategy is to adopt a two-step classification approach based on the detection of "confident abnormal respiration." In the first step, hidden Markov models and bigram models are used for acoustic features and the occurrence of acoustic segments in each abnormal respiratory period, respectively, to calculate two kinds of stochastic likelihoods: the highest likelihood for a segment sequence to be abnormal respiration and the likelihood for normal respiration. In the second step, the patients are identified on the basis of the detection of confident abnormal respiration, which is when difference between these two likelihoods is larger than a predefined threshold. Our strategy achieved the highest classification rate of $88.7 \%$ between healthy subjects and patients among three basic classification strategies, which shows the validity of our approach.
\end{abstract} model

Index Terms - lung sound, adventitious sound, acoustic

\section{INTRODUCTION}

The auscultation of lung sounds is one of the most popular medical examination methods for identifying respiratory illnesses. This is because abnormal respiratory sounds usually appear in patients. Sounds such as wheezes are caused by abnormalities in the lungs and bronchial tubes; they are termed as "adventitious sounds." The intensity and distinctness of adventitious sounds are very low. Hence, these sounds could be mistaken for environmental noises, which are frequently mixed in with lung sounds. To detect adventitious sounds correctly, in-depth experience and knowledge possessed by doctors are required. Then, automated diagnosis of pulmonary emphysema through the detection of abnormal respiratory sounds by using a stethoscope at home would be beneficial because appropriate medical treatment could be administered to patients at an early stage.

Several studies have been conducted on acoustic analysis of respiratory sounds for the detection of specific adventitious sounds [1-4]. These studies were not, however, aimed at developing devices to identify respiratory illness at home; instead, they were aimed at assisting doctors in hospitals to make diagnoses.

The objective of our study is to develop a home-use device for identifying the respiratory illness by detecting abnormal respiratory sounds in lung sounds. For this purpose, we collected lung sound data from patients and healthy subjects and then developed a classification procedure for distinguishing between normal and abnormal respiratory sounds on the basis of a maximum likelihood approach using hidden Markov models (HMMs) [5,6]. To calculate the likelihood of normal/abnormal respiration, we assumed that one section of each respiratory period consisted of a time series of acoustic segments that express specific acoustic features such as adventitious sounds. In these preliminary classification experiments, we used deterministic rules or a segment bigram to express the occurrence of acoustic segments in abnormal respiratory sounds. Classification results from these experiments indicated that the stochastic method related to acoustic HMMs and segment $n$-grams shows promise.

In our study, we attempted to design a classification system to differentiate between a healthy subject and patient with pulmonary emphysema by the detection of adventitious sounds. Due to the various kinds of adventitious sounds and noises at auscultation, it is difficult to detect adventitious sounds in during respiration with high accuracy. This problem leads to low classification performance between healthy subjects and patients.

To address this problem, we propose a robust classification strategy based on the detection of "confident abnormalrespiration.” Our classification consists of a two-step process. In the first step, for each respiration in a lung sound sample, the highest stochastic likelihoods to be an abnormal respiration and a normal respiration are each calculated. In the second step, for each respiration, the difference between these likelihoods is calculated. If this difference is larger than the predefined threshold, this respiration can be confidently regarded to be normal or abnormal. If there is at least one confident abnormal respiration in the tested lung sound sample, our system regards the test subject to be a patient with pulmonary emphysema. Next, we used the predefined threshold identified by polynomial curve fitting of the histogram of the difference values. The validity of this method for determining the threshold is also discussed in this paper.

\section{LUNG SOUND DATA}

\subsection{Recording conditions}

We recorded 112 lung sound samples from 101 patients with pulmonary emphysema and 39 samples from 39 healthy subjects. In the recording at auscultation, an electronic stethoscope incorporating a piezoelectric microphone was used. We used the second intercostal space on the subjects' front right as the recording point.

Each lung sound sample consisted of successive respiratory phase segments, and the average number of respiratory segments 
was 8. These segments were labeled according to the respiratory phase (inspiratory or expiratory), diagnostic state (normal or abnormal), and subject's states (healthy or patient). The subject's state was identified by a doctor using not only auscultation but also many other medical conditions. In the recording using the stethoscope, mixing with noises is inevitable, and consequently $79.6 \%$ of all respiration included some noises from the stethoscope or internal organs. In this paper, the meaning and abbreviation for each kind of respiration is as follows:

- Abnormal respiration from patient subjects (AP): respiration that contains obvious adventitious sounds.

- Abnormal respiration from healthy subjects (AH): respiration that contains noises from internal organs similar to adventitious sounds. The number of this type was rather small.

- Normal respiration from healthy subjects $(\mathrm{NH})$ : respiration that contains neither adventitious sounds nor adventitious-like noises.

- Normal respiration from patient subjects (NP): respiration that does not contain obvious adventitious sounds but sometimes contains adventitious-like noises.

Each of our lung sound data from patients contained one or more adventitious sounds; the detection of abnormal respiration from patient subjects (AP) is then necessary to identify the patients. Respiration data concerning AP and $\mathrm{NH}$ were used to confirm the ability to classify the adventitious sounds of patients and normal respiratory sounds of healthy subjects, and all respirations were used to classify the patients and healthy subjects. The number of respiratory segments is listed in Table 1.

Table 1. Number of respiratory periods

\begin{tabular}{c|c|c}
\hline Respiration & Patients & Healthy subjects \\
\hline \hline Normal & $451(\mathrm{NP})$ & $348(\mathrm{NH})$ \\
\hline Abnormal & $371(\mathrm{AP})$ & $33(\mathrm{AH})$ \\
\hline
\end{tabular}

\subsection{Hand labeling of acoustic segments}

We considered an abnormal respiratory period to be composed of successive acoustic segments. In order to detect the adventitious sounds of patient subjects by using a stochastic method, we defined the segments according to their acoustic features and assigned a symbol to each segment. The respiratory data were labeled by hand with the symbols by two experts and one doctor.

Suppose a respiratory period $W$ comprises $N$ segments: let the $i$-th segment be $w_{i}(1 \leq i \leq N)$. Then,

$$
W=w_{1} w_{2} \cdots w_{i} \cdots w_{N} .
$$

In our data, one abnormal respiratory period comprises several segments, and one normal respiratory period comprises one breath segment $(N=1)$. In this study, each adventitious sound was presented by using a continuous or discontinuous sound segment; the segment sequence of an abnormal respiratory period thus consisted of one of two types of segments and respiratory-sound segments not including adventitious sounds. Typical examples of continuous sound segments are coarse crackle, fine crackle, and pleural friction rub. Types such as rhonchus or wheezing sounds belong to the discontinuous segments. These classes of adventitious sounds were designed based on classification by the American Thoracic Society (ATS).

\section{CLASSIFICATION PROCEDURE}

\subsection{Abnormal respiratory detection}

Let the occurrence probability of the segment sequence $W$ in respiration be $P(W)$ :

$$
P(W)=P\left(w_{1} w_{2} \cdots w_{i} \cdots w_{N}\right) .
$$

In this study, we used a segmental bigram to calculate $P(W)$ :

$$
P(W) \approx \sum_{i=2}^{N} P\left(w_{i} \mid w_{i-1}\right) .
$$

The total likelihood composed of the acoustic likelihood derived from HMMs and the segmental sequence likelihood derived from equation (3) is calculated by using a weight factor $\alpha$. The derived diagnostic state (normal/abnormal) for respiratory input that gives the segment (sequence) $\hat{W}$ with the highest likelihood $P(\hat{W} \mid X)$ is given below.

$$
\underset{W}{\arg \max } P(W \mid X)=\underset{W}{\arg \max }(\alpha \log P(W)+\log P(X \mid W)) .
$$

where $X$ is the respiratory input and $P(X \mid W)$ is the acoustic likelihood. The weight factor $\alpha$ controls the contribution of the bigram. If $\alpha$ is equal to 0 , classification is carried out by using acoustic HMMs and the deterministic connection rules of the acoustic segments. In this study, the value of $\alpha$ was experimentally acquired.

\subsection{Criteria of patient detection}

The classification between healthy and patient subjects was carried out by using two likelihoods obtained using $P(\hat{W} \mid X)$ for normal and abnormal respiration. The "confident abnormal respiration" is defined as follows: if the difference between the likelihood $Q$ for the normal respiration candidate $W_{\text {normal }}$ and the likelihood for abnormal respiration candidate $\hat{W}_{\text {abnormal }}$ is larger than the threshold $T h$ for respiratory input, we regard this test respiration $X$ to be abnormal with confidence. That is,

$$
Q(X)=\log P\left(\hat{W}_{\text {abnormal }} \mid X\right)-\log P\left(W_{\text {normal }} \mid X\right)>\text { Th. } .
$$

A lung sound sample consists of several respiratory periods. Then, if our system detects one or more confident abnormal respiration periods in a test lung sound sample, it classifies the result to be a "patient subject."

Setting an adequate threshold Th is also a difficult problem. To cope with this issue, we prepared two kinds of histograms for the difference value $Q$ : one for abnormal samples that were classified correctly, and the other for abnormal samples that were mistakenly classified as normal. We carried out fitting by using a fourth-polynomial curve for each histogram, and we set the crosspoint of these two curves as the threshold Th. In our experiments, two kinds of thresholds for inspiration and expiration were used.

\subsection{Patient detection procedure}

The architecture of our classification system is shown in Figure 1. The system comprises two steps: first, abnormal and normal respirations are classified by calculating the stochastic likelihoods, and second, healthy and patient subjects are classified.

In the first step, we used HMMs for acoustic spectral features and bigram models for the occurrence of acoustic segments in each respiratory period. This step comprised a training process and a test process. In the training process, acoustic HMMs for 


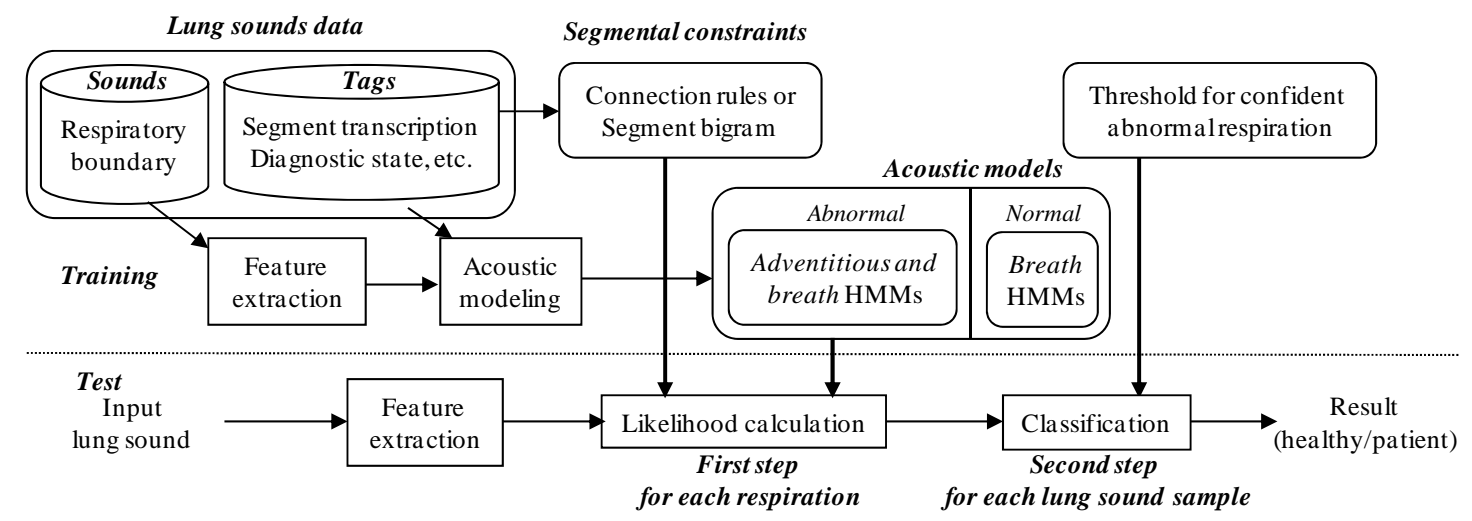

Figure 1. Classification procedure for distinguishing between healthy and patient subjects

each kind of segment were generated for each respiratory phase. Thus, we prepared two models for inspiration and expiration. With regard to abnormal respiration, acoustic models corresponding to each acoustic segment type were generated for inspiration/expiration. Segment bigrams with reference to the occurrence sequences of the segments in abnormal respiration were also estimated according to the segment labels. Rules concerning occurrence sequences of the acoustic segments in the abnormal respiration were also represented by hand. In the test process, the two acoustic likelihoods of an input respiration were calculated by using the trained HMMs and segment bigrams. One likelihood is for the segment sequence with the highest likelihood of abnormal respiration, and the other is for normal respiration.

In the second step, based on the detection of confident abnormal respiration in the test lung sound, the test subject is determined to be ill or healthy, as described in section 3.2.

\section{EVALUATION EXPERIMENTS}

We conducted two types of classification tests to evaluate our proposed strategy. First, we classified between abnormal and normal respiration by using the first step. The lung sound data were sampled at $10 \mathrm{kHz}$. Every $10 \mathrm{~ms}$, a vector of 5 mel-warped cepstral coefficients and power was computed using a 25-ms Hamming window. Acoustic models for normal respiration were generated using the respiratory sounds from healthy subjects $(\mathrm{NH}$ in section 2.1), and models for abnormal respiration were generated using the sounds from patients (AP). HMMs with 3 states and 2 Gaussian probability density functions (2-mixture of PDFs) were used. In our experiments, we assumed that the respiratory phase and respiratory boundaries are known. As such, if the test sample was expiratory, acoustic models generated with expiratory sounds were used for respiratory classification.

\subsection{Performance of classification between abnormal and normal respiration}

To confirm the detection performance of adventitious sounds in the first step, a classification experiment between abnormal respiration for patients and normal respiration for healthy subjects was carried out using the deterministic connection rules or segmental bigrams, respectively. Evaluation samples were abnormal respirations from patients (AP) and normal respirations from healthy subjects (NH). We performed a leave-one-out cross validation on these samples. In addition, samples recorded from the same subject as the test sample were excluded in the training process so that our experiments would be subject-independent. The classification results are shown in Table 2 . The recall rate of the abnormal respiration was $93.7 \%$, and that of the normal respiration was $89.2 \%$ when the segment bigram was used. The average classification rate weighted with the data amount is indicated as “Average.” This result shows that each method using the detection rules or segment bigram achieved a good performance, and the latter method achieved a slightly better performance.

Furthermore, we carried out recognition experiments for abnormal respiration from healthy subjects $(\mathrm{AH})$ and for normal respiration from patients (NP). The recognition results are shown in Table 3. This result shows that identification of NP that does not include obvious adventitious sounds to be normal respiration was difficult; however, this problem is not insurmountable for identifying respiratory illnesses by using our classification strategy.

Table 2: Classification rates of abnormal respiration for patients and normal respiration for healthy subjects [\%]

\begin{tabular}{l|c|c|c}
\hline $\begin{array}{l}\text { Segment } \\
\text { connection }\end{array}$ & $\begin{array}{c}\text { Patient, } \\
\text { abnormal(AP) }\end{array}$ & $\begin{array}{c}\text { Healthy, } \\
\text { normal(NH) }\end{array}$ & $\begin{array}{c}\text { Averag } \\
\mathrm{e}\end{array}$ \\
\hline \hline Deterministic rules & 92.2 & 84.9 & 88.5 \\
\hline Segment bigram & 93.7 & 89.2 & 91.4 \\
\hline
\end{tabular}

Table 3: Recognition results for abnormal respiration of healthy subjects and normal respiration of patient subjects [\%]

\begin{tabular}{c|c|c}
\hline Segment connection & $\begin{array}{c}\text { Healthy, } \\
\text { abnormal (AH) }\end{array}$ & $\begin{array}{c}\text { Patient, } \\
\text { normal (NP) }\end{array}$ \\
\hline \hline Deterministic rules & 97 & 59.9 \\
\hline Segment bigram & 97 & 62.1 \\
\hline
\end{tabular}

\subsection{Effect of confident abnormal respiration}

One of the main characteristics of our proposed strategy is to find confident abnormal respiration to detect patients by using the thresholds derived from fourth polynomial curve fitting. To confirm the validity of this threshold, we investigated the recall rates of abnormal respiration for the results whose difference value $Q$ was over and under the threshold. Table 4 shows the investigation results. For both experiments using the deterministic rules and segment bigram, the recall rates for the samples $Q>T h$ were far better than those of samples $Q<T h$. This result indicates the promising potential of using confident abnormal respiration. 
Table 4: Recall rates of abnormal respiration for patients [\%]

\begin{tabular}{c|c|c}
\hline Segment connection & $Q>T h$ & $Q<T h$ \\
\hline \hline Deterministic rules & 93.9 & 84.2 \\
\hline Segment bigram & 96.5 & 84.8 \\
\hline
\end{tabular}

\subsection{Classification between healthy and patient subjects}

The classification experiments were carried out by using 112 lung sounds samples. To evaluate our proposed strategy, three criteria to identify a patient were compared: (C1) detecting one or more confident abnormal respirations; (C2) detecting abnormal respiration, i.e., if a lung sound data sample includes at least one abnormal respiration, the subject is regarded as patient; (C3) comparing results of two total likelihoods for all respiratory periods of normal respiration $\sum_{i} \log P\left(\hat{W}_{\text {abnormal }} \mid X_{i}\right)$ and for abnormal respiration $\sum_{i} \log P\left(W_{\text {normal }} \mid X_{i}\right)$. If the total likelihood for abnormal respiration is larger than that for normal respiration, the subject is regarded as a patient.

Table 5 shows the classification performance using each classification criterion. The proposed strategy with confident abnormal respiration detection C1 achieved the highest performance among the three criteria. We believe that this result indicates the feasibility of automatic detection of patients with pulmonary emphysema by using lung sounds.

Table 5: Classification performance between healthy subjects and patient subjects (C1: confident abnormal respiration, C2: abnormal respiration, C3: total likelihoods) [\%]

\begin{tabular}{c|c|c|c|c}
\hline $\begin{array}{c}\text { Segment } \\
\text { connection }\end{array}$ & $\begin{array}{c}\text { Classification } \\
\text { criterion }\end{array}$ & $\begin{array}{c}\text { Healthy } \\
\text { subject }\end{array}$ & $\begin{array}{c}\text { Patient } \\
\text { subject }\end{array}$ & $\begin{array}{l}\text { Avera } \\
\text { ge }\end{array}$ \\
\hline \hline \multirow{3}{*}{$\begin{array}{c}\text { Deterministic } \\
\text { rules }\end{array}$} & C1 (proposed) & 95 & 86 & 87.4 \\
\cline { 2 - 5 } & C2 & 18 & 95 & 75.4 \\
\cline { 2 - 5 } & C3 & 77 & 73 & 74.2 \\
\hline \multirow{3}{*}{$\begin{array}{c}\text { Segment } \\
\text { bigram }\end{array}$} & C1 (proposed) & 95 & 88 & 88.7 \\
\cline { 2 - 5 } & C2 & 18 & 95 & 75.4 \\
\cline { 2 - 5 } & C3 & 77 & 74 & 74.8 \\
\hline
\end{tabular}

\subsection{Validity of difference value for patient detection}

In the previous experiments, the difference value $T h$ that was derived from the fitting of the fourth-polynomial curve was used (312 for inspiration and 301 for expiration). To confirm the validity of this obtained value $T h$, classification experiments between healthy and patient subjects were carried out using several thresholds. The recall rates of healthy subjects and patients subjects, and the classification rate of all subjects are shown in Figure 2; the horizontal axis indicates the threshold, and the vertical axis indicates the performance. The highest classification performance among these thresholds was $89.4 \%(T h=213)$, which is slightly superior to the figure shown in Table 5 (88.7\%). This result shows that the difference value derived from the polynomial fitting gave a good performance; however, there is still room to improve the performance by selecting better thresholds.

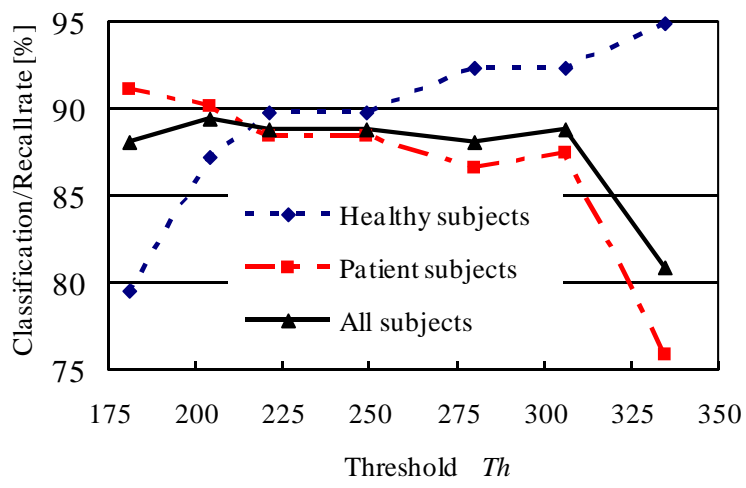

Figure 2: Classification rate for each threshold

\section{CONCLUSIONS}

This paper proposes a procedure for discriminating between healthy subjects and patients with abnormal respiration based on the detection of "confident abnormal respiration." In our procedure, two stochastic likelihoods concerning the best abnormal and normal respiration candidates play an important role. These likelihoods were calculated by using HMMs for acoustic spectral features and bigram models for the occurrence of acoustic segments in each respiratory period. By using the difference value between these two likelihoods, a robust classification system for healthy and patient subjects was constructed. According to the classification experiments, our detection strategy achieved the best classification rate among three basic patient-detection criteria, which shows its effectiveness.

Concerning the threshold of the difference value to detect confident abnormal respiration, our experiments indicated that there is still room for improving the performance by setting a more appropriate threshold. Devising a method to capture the difference threshold is a subject for future work.

\section{REFERENCES}

[1] Y.P. Kahya, S. Yere and O. Cerid, "A wavelet-based instrument for detection of crackles in pulmonary sounds," Proc. of IEEE EMBS, pp.3175-3178, 2001.

[2] M. Bahoura and X. Lu, "Separation of crackles from vesicular sounds using wavelet packet transform," Proc. of IEEE ICASSP, II, pp. 1076-1079, 2006.

[3] S.A. Taplidou and L.J. Hadjileontiadis, "Wheeze detection based on time-frequency analysis of breath sounds," Computers in Biology and Medicine, Vol. 37, pp.1073-1083, 2007.

[4] A. Marshall and S. Boussakta, "Signal analysis of medical acoustic sounds with applications to chest medicine," Journal of the Franklin Institute, Vol. 344, pp.230-242, 2007.

[5] S. Matsunaga, et al., "Classification between normal and abnormal respiratory sounds based on maximum likelihood approach,” Proc. of IEEE ICASSP, pp. 517-520, 2009.

[6] H. Yamamoto, et al., "Classification between normal and abnormal respiratory sounds based on stochastic approach,” Proc. of ICA, 2010. 